\title{
Le SMB est mort, vive le SMB!
}

\section{Hans Stalder}

Prof. Dr méd., membre de la rédaction

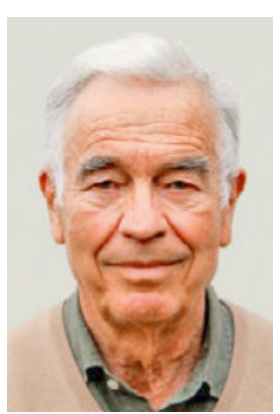

Vu la rapidité des progrès techniques et thérapeutiques, il est devenu important qu'il y ait des structures indépendantes pour apprécier leur pertinence tant pour les médecins (pour générer des guidelines) que pour les responsables du système de santé (pour apprécier leur valeur ajoutée au niveau économique). Ainsi, dès la fin du siècle dernier, des institutions ont été créées dans différents pays, dont la plus reconnue est le National Institute for Health and Care Excellence (NICE) en Angleterre. Des structures similaires existent en France (Haute Autorité de Santé, HAS), en Allemagne (Institut für Qualität und Wirtschaftlichkeit im Gesundheitswesen, IQWiG) et ailleurs en Europe, toutes associées dans l'European Network for Health Technology Assessment (EUnetHTA). Les caractéristiques principales de ces structures sont leur compétence scientifique, leur indépendance, aussi bien de l'industrie et des caisses-maladie que des corporations médicales, et leur transparence. Leurs procédures, respectant des standards internationaux, comportent trois volets: évaluation (assessment) ou Health technology Assessment (HTA), appréciation (appraisal) et décision. L'évaluation consiste en un examen systématique des preuves scientifiques disponibles, en particulier du point de vue de l'innocuité et de l'efficacité, ainsi que de l'utilité supplémentaire par rapport aux alternatives existantes. L'appréciation tient compte des aspects juridiques, éthiques et sociaux et formule des recommandations après consultation des différentes parties intéressées (médecins, industrie, caisses-maladie, patients...). L'évaluation et l'appréciation doivent être indépendantes de l'institution fâ̂tière qui prendra les décisions.

En Suisse, l'initiative d'une telle structure a été lancée par la Direction de la santé du canton de Zurich en 2008 avec le projet pilote «Medical Board» «pour contribuer à garantir l'efficacité, l'adéquation et l'économicité des traitements médicaux exigées dans la Loi sur l'assurance-maladie». En septembre 2009, la Conférence suisse des directeurs cantonaux de la santé (CDS) s'y est associée. En 2010, la FMH et l'Académie suisse des sciences médicales (ASSM) ont accepté d'assumer la coresponsabilité du Medical Board avec la CDS et en février 2011 ces trois institutions ont créé le Swiss Medical Board (SMB).

Le SMB a fait preuve de passablement de courage, mais aussi d'une certaine naïveté dans l'élaboration des recommandations. Certaines ont provoqué des réactions souvent violentes, mais en partie justifiées. Aussi le soutien financier se faisait rare. De plus, pour faire barrage à certaines décisions potentiellement préjudiciables du SMB, les caisses-maladie et l'industrie ont fondé le SwissHTA.

Pour assurer son financement, le SMB a fusionné avec le SwissHTA en janvier 2015. Le SMB est actuellement dirigé par un nouvel organe responsable qui est composé de représentants de la CDS, l'ASSM, la Principauté du Liechtenstein, Curafutura, Interpharma et Santésuisse. Pour rester indépendante face aux activités HTA, la FMH (mais pas l'ASSM) en est sortie. Si l'organe responsable n'exerce toujours aucune influence sur les contenus de l'évaluation et de l'appréciation ainsi que sur l'attribution des mandats, il décide du développement stratégique, de la définition des organes opérationnels, de la sélection des thèmes, de la communication et de la publication des rapports et des mesures pour la mise en œuvre des recommandations. Les membres de l'organe responsable, en particulier l'industrie et les caisses-maladie, peuvent donc bloquer la publication des décisions de l'évaluation et de l'appréciation si celles-ci ne leur plaisent pas. De ce fait, le SMB a vendu son âme en abandonnant les principes les plus importants qui caractérisent une telle structure: sa transparence et son indépendance, et donc sa crédibilité.

Pour assurer l'indépendance des HTA, l'organe de financement ne peut être qu'étatique, comme c'est le cas dans les autres pays d'Europe. Comme déjà suggéré lors de l'initiative zurichoise, le Conseil fédéral semble enfin agir dans ce sens. L'examen systématique et régulier de l'utilité des prestations médicales est une priorité de sa stratégie "Santé2020». Espérons que ses intentions ne seront pas uniquement d'ordre économique - réduire les coûts de la santé -, mais aussi d'augmenter la qualité des soins afin que renaisse un SMB fonctionnant enfin selon les règles de l'art. 